\title{
Anticancer Activity of Lichen Extract (Usnea sp.) Based Synthesized Ag@ZnO Bimetallic Nanocomposite ${ }^{\dagger}$
}

\author{
Yakup Isik ${ }^{1}$, Gokhan Unal ${ }^{2}$, Fatih Dogan Koca ${ }^{3}$ and Mehmet Gokhan Halici 1,* \\ 1 Department of Biology, Faculty of Science, Erciyes University, Kayseri 38039, Turkey; \\ yakup.isiq@gmail.com \\ 2 Department of Pharmacology, Faculty of Pharmacy, Erciyes University, Kayseri 38039, Turkey; \\ gokhanunal@erciyes.edu.tr \\ 3 Department of Aquatic Animal and Diseases, Faculty of Veterinary Medicine, Erciyes University, \\ Kayseri 38039, Turkey; fatihdkoca@erciyes.edu.tr \\ * Correspondence: mghalici@erciyes.edu.tr \\ + Presented at the 3rd International conference on Natural Products for Cancer Prevention and Therapy, \\ Kayseri, Turkey, 18-20 December 2019.
}

Published: 3 March 2020

\begin{abstract}
Bimetallic nanoparticles (NP) have improved properties such as antimicrobial, anticancer and photocatalytic activity due to the synergistic effects of their components. Unmet needs of the current cancer treatments encourage researchers for the new treatment approaches especially natural products. In this study, we aimed to investigate the antiproliferative effects of Ag@ZnO NP on the SH-SY5Y human neuroblastoma cell line. Ag@ZnO NP was dispersed in distilled water via ultrasonication and diluted with the medium as the 5, 10, 25, 50, 100, 200 and $400 \mu \mathrm{g} / \mathrm{mL}$ final concentrations. The cell viabilities were investigated by MTT test at 24th hours of Ag@ZnO NP treatments. In MTT test, the lower concentrations of Ag@ZnO NP $(5,10,25 \mu \mathrm{g} / \mathrm{mL})$ increased the cell viability $(p<0.01, p<0.01$ and $p<0.05$, respectively) whereas it was markedly decreased ( $p<$ $0.001)$ the cell viabilities at higher concentrations $(100,200$ and $400 \mathrm{ug} / \mathrm{ml})$ compared with the control group. Our results showed that Ag@ZnO NP has antiproliferative effects on SH-SY5Y cells whereas it increases cell proliferation in the low dose. The effects in the low dose may depend on its activation of compensatory mechanisms in the cell. We have thought that it is worthwhile to research its anticancer potential on the different cancer cell lines.
\end{abstract}

Keywords: silver and zinc oxide nanoparticles; SH-SY5Y; cytotoxicity; cancer

(C) 2020 by the authors. Licensee MDPI, Basel, Switzerland. This article is an open access article distributed under the terms and conditions of the Creative Commons Attribution (CC BY) license (http://creativecommons.org/licenses/by/4.0/). 